\title{
HADAMARD CONVEXITY AND MULTIPLICITY AND LOCATION OF ZEROS
}

\author{
FARUK F. ABI-KHUZAM
}

\begin{abstract}
We consider certain questions arising from a paper of Hayman concerning quantitative versions of the Hadamard three-circle theorem for entire functions. If $b(r)$ denotes the second derivative of $\log M(r)$ with respect to $\log r$, the principal contributions of this work are (i) a characterization of those entire $f$ with nonnegative Maclaurin coefficients for which $\lim \sup b(r)=\frac{1}{4}$ and (ii) some exploration of the relationship between multiple zeros of $f$ and the growth of $b(r)$.
\end{abstract}

\section{INTRODUCTION}

Let $f$ be an entire function and put

$$
b(r)=b(r ; f)=d^{2} \log M(r) / d(\log r)^{2}
$$

where $M(r)=M(r ; f)=\sup _{|z|=r}|f(z)|$ is the maximum modulus of $f$. The simplest result of this paper is the following:

Theorem. If $f$ has nonnegative Maclaurin coefficients and satisfies

$$
\limsup _{r \rightarrow \infty} b(r)=\frac{1}{4}
$$

then all but a finite number of the zeros of $f$ are simple and lie on the negative real axis. Furthermore, if $r_{n}$ is the modulus of the nth zero then

$$
r_{n+1} / r_{n} \rightarrow \infty \text { as } n \rightarrow \infty \text {. }
$$

This theorem puts in sharp form an earlier result [1] where the zeros were shown to lie in arbitrarily small angles containing the negative real axis.

The study of the growth of $b(r)$ was initiated in 1966 by Hayman [3] who showed that the classical estimate $b(r) \geq 0$ obtained from Hadamard's three-circle theorem could be improved under certain conditions. Hayman showed that there exists a positive absolute constant $A_{0}>0.180$ such that limsup $b(r) \geq A_{0}$ for every transcendental entire function $f$. In 1973, Kjelle-

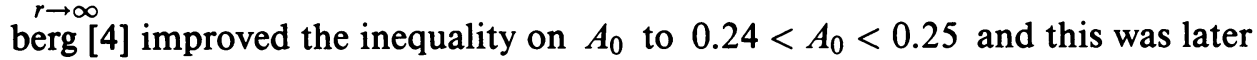
improved to $0.24<A_{0}<0.247$ by Hilditch [5]. In the special case where $f$

Received by the editors November 12, 1993 and, in revised form June 21, 1994; originally communicated to the Proceedings of the AMS by Albert Baernstein.

1991 Mathematics Subject Classification. Primary 30D20, 30D15; Secondary 30D35. 
has nonnegative Maclaurin coefficients, Boichuck and Gol'dberg [2] obtained $A_{0}=0.25$ which is a sharp result for this class of functions. The best possible value of $A_{0}$ in the class of all transcendental entire functions is as yet unknown. The fact that the growth of $b(r)$ is related to the distribution of values of $f$ was first pointed out in [1] where, also, another proof of the Boichuck-Gol'dberg result was obtained. Here we go a little further by investigating the relationship between the growth of $b(r)$ and the multiple zeros of $f$ or, more generally, zeros having equal moduli. It turns out that there is an absolute constant $2 A_{1}$, a sort of an "impact parameter", such that if $\limsup b(r)<2 A_{1}$ and $f$ has only real coefficients, then all but a finite number of the zeros of $f$ must be real and simple. Since $2 A_{1}>0.36$, the theorem stated above follows from this and the positivity of the coefficients. The exact value 0.25 in (2) is needed to get (3).

The most concise way of stating our results requires the use of Jacobian elliptic functions, and we start by summarizing what is needed from their theory [6].

We set $q=e^{\pi i \tau}$ where $\operatorname{Im} \tau>0$ and define the theta function $\theta$ by

$$
\theta(z)=\theta_{4}(z)=\sum_{n=-\infty}^{\infty}(-1)^{n} q^{n^{2}} e^{2 i n z}
$$

The other theta functions $\theta_{1}, \theta_{2}$ and $\theta_{3}$ are defined in terms of $\theta_{4}$ and we have the product representations $[6, \mathrm{p} .470]$

$$
\theta_{1}(z)=2 G q^{1 / 4} \sin z \prod_{n=1}^{\infty}\left(1-q^{2 n} e^{2 i z}\right)\left(1-q^{2 n} e^{-2 i z}\right)
$$

$$
\theta_{2}(z)=2 G q^{1 / 4} \cos z \prod_{n=1}^{\infty}\left(1+q^{2 n} e^{2 i z}\right)\left(1+q^{2 n} e^{-2 i z}\right)
$$

where $G$ is a constant whose precise value need not concern us at the moment. The Jacobian elliptic functions may be defined [6, p. 492] by

$$
s n(z, k)=\frac{\theta_{3}(0)}{\theta_{2}(0)} \cdot \frac{\theta_{1}\left(z / \theta_{3}^{2}(0)\right)}{\theta_{4}\left(z / \theta_{3}^{2}(0)\right)}
$$

$$
c n(z, k)=\frac{\theta_{4}(0)}{\theta_{2}(0)} \cdot \frac{\theta_{2}\left(z / \theta_{3}^{2}(0)\right)}{\theta_{4}\left(z / \theta_{3}^{2}(0)\right)} .
$$

Here $k$ is a constant (called the modulus) defined in terms of $q$ and confined, when $q$ is real, to the interval $(0,1)$. Associated to $k$ are constants $K, k^{\prime}$, $K^{\prime}$ that may conveniently be defined, at least when $k \in(0,1)$, by

$$
\begin{gathered}
K=\int_{0}^{\pi / 2} \frac{d t}{\sqrt{1-k^{2} \sin ^{2} t}}, \quad k^{\prime 2}+k^{2}=1\left(k^{\prime}>0\right), \\
K^{\prime}=\int_{0}^{\pi / 2} \frac{d t}{\sqrt{1-k^{\prime 2} \sin ^{2} t}},
\end{gathered}
$$


and one has the relations [6, p. 479]

$$
K^{\prime}=\frac{K}{\pi} \log \left(\frac{1}{q}\right), \quad k^{\prime 1 / 2}=\frac{\theta_{4}(0)}{\theta_{3}(0)}, \quad \theta_{3}^{2}(0)=\frac{2 K}{\pi}, \quad s n\left(K^{\prime}, k^{\prime}\right)=1 .
$$

From (6) and (8) one obtains

$$
\frac{\theta_{1}(\pi z / 2 K)}{\theta_{2}(\pi z / 2 K)}=k^{\prime 1 / 2} \cdot \frac{s n(z, k)}{c n(z, k)} .
$$

Simple explicit values for $k^{\prime}$ may be obtained for certain given values of $q$ or $K / K^{\prime}$. Thus one has, for example, the following table [6, p. 525]

\begin{tabular}{|c|c|c|c|c|}
\hline$K / K^{\prime}$ & 1 & $\sqrt{2}$ & $\sqrt{3}$ & 2 \\
\hline$k^{\prime}$ & $\frac{1}{\sqrt{2}}$ & $\sqrt{2}-1$ & $\frac{1}{\sqrt{2}(\sqrt{3}+1)}$ & $(\sqrt{2}-1)^{2}$ \\
\hline
\end{tabular}

In general, however, we have [6, p. 479]

$$
k^{\prime 1 / 2}=\frac{1-2 q+2 q^{4}+2 q^{9}+\cdots}{1+2 q+2 q^{4}+2 q^{9}+\cdots}=\prod_{n=1}^{\infty}\left(\frac{1-q^{2 n-1}}{1+q^{2 n-1}}\right)^{2}
$$

and the middle expression in (10) is convenient for the calculation of $k^{\prime}$.

Let $\alpha>0$ and put $q=e^{-\pi^{2} / \alpha}$, so that $k^{\prime}$ becomes a function of $\alpha$ and $K^{\prime} / K=\pi / \alpha$. Define

$$
A_{1}(\alpha)=\frac{-8}{\alpha^{2}} \log k^{\prime 1 / 2}=\frac{-8}{\alpha^{2}} \log k^{\prime}(\alpha)^{1 / 2} .
$$

Then $A_{1}(\alpha)>0$ and it can be shown that $A_{1}(\alpha) \rightarrow 0$ as $\alpha \rightarrow 0+$ or $\infty$. So $A_{1}(\alpha)$ has an absolute maximum on $(0, \infty)$ at some point $\alpha_{0}$. The table gives $A_{1}(\pi \sqrt{2})=\frac{-2}{\pi^{2}} \log (\sqrt{2}-1)=A_{1}(2 \pi)$ and so a critical value of $A_{1}$ exists between $\pi \sqrt{2}$ and $2 \pi$. At any rate we have $A_{1}\left(\alpha_{0}\right) \geq A_{1}(\pi \sqrt{3})=\frac{4}{3 \pi^{2}} \log (\sqrt{6}+\sqrt{2}) \simeq$ 0.183 .

Throughout this paper we shall take for $q$ the value $e^{-\pi^{2} / \alpha_{0}}$ where, as mentioned above,

$$
A_{1}\left(\alpha_{0}\right)=\max _{(0, \infty)} A_{1}(\alpha)=A_{1} .
$$

The "impact parameter" referred to earlier in this introduction will be the constant $2 A_{1}$, and the above discussion shows that $2 A_{1}>0.365$.

\section{Statement of Results}

For $r_{0}>0$ and $\alpha_{0}$ defined by (12) we shall use the notation

$$
D\left(r_{0}, \alpha_{0}\right)=\left\{z: r_{0} \exp \left(-\frac{1}{2} \alpha_{0}\right)<|z|<r_{0} \exp \left(\frac{1}{2} \alpha_{0}\right)\right\}
$$

and

$$
I\left(r_{0}, \alpha_{0}\right)=\left\{r: r_{0} \exp \left(-\frac{1}{2} \alpha_{0}\right)<r<r_{0} \exp \left(\frac{1}{2} \alpha_{0}\right)\right\}
$$


Theorem 1. Let $f(z)$ be regular in $D\left(r_{0}, \alpha_{0}\right)$.

(i) If $f\left(z_{1}\right)=0$ for some point $z_{1}$ satisfying $\left|z_{1}\right|=r_{0}$, then there exists $r$ in $I\left(r_{0}, \alpha_{0}\right)$ such that $b(r) \geq A_{1}$ where $A_{1}$ is defined in (12).

(ii) If $f\left(z_{1}\right)=f\left(z_{2}\right)=0$ where $\left|z_{1}\right|=\left|z_{2}\right|=r_{0}$ there exists $r \in I\left(r_{0}, \alpha_{0}\right)$ such that $b(r) \geq 2 A_{1}$ where $A_{1}$ is defined by (12).

Corollary 1. If $f$ is a transcendental entire function then

$$
\underset{r \rightarrow \infty}{\limsup _{r \rightarrow \infty}} b(r) \geq A_{1} \text {. }
$$

Corollary 2. Let $f(z)=\sum_{k=0}^{\infty} a_{k} z^{k}$ be a transcendental entire function and assume that

$$
\limsup _{r \rightarrow \infty} b(r)<2 A_{1} \text {. }
$$

Then all but a finite number of the zeros of $f$ have distinct moduli, in particular,

(i) if all the coefficients of $f$ are real, then all but a finite number of the zeros of $f$ are simple and real;

(ii) if all the coefficients of $f$ are nonnegative, then all but a finite number of the zeros of $f$ are simple and lie on the negative real axis.

Theorem 2. Let $f(z)=\sum_{k=0}^{\infty} a_{k} z^{k}$ be a transcendental entire function whose coefficients $a_{k}$ are nonnegative. If $b(r)$ is bounded, then $\limsup _{r \rightarrow \infty} b(r)=0.25$ if and only if all but a finite number of zeros $\left\{z_{n}\right\}$ of $f$ are simple, negative and satisfy $r_{n+1} / r_{n} \rightarrow \infty$ as $n \rightarrow \infty$ where $r_{n}=\left|z_{n}\right|$.

We do not know whether equality can hold in relation (2.1), but we can have limsup $b(r)=2 A_{1}$. This raises the question of determining the extremal functions. In order to do so let us write $\omega(r)$ for any argument at which the maximum modulus is achieved. That is $M(r)=\left|f\left(r e^{i \omega(r)}\right)\right|$. If $z_{n}$ is the $n$th zero of $f$ we write $z_{n}=r_{n} e^{i \theta_{n}}$.

Theorem 3. Let $f$ be an entire function and let $\delta, 0<\delta \leq \pi$, be given. then

(i) If there are infinitely many zeros $z_{n}=r_{n} e^{i \theta_{n}}$ satisfying $\left|\theta_{n}-\omega\left(r_{n}\right)\right| \leq \delta$

$$
\limsup _{r \rightarrow \infty} b(r) \geq A_{1}-\frac{8}{\alpha_{0}^{2}} \log \left|s n\left(\frac{K^{\prime} \delta}{\pi}, k^{\prime}\right)\right| .
$$

(ii) If there are infinitely many pairs of zeros $z_{n}=r_{n} e^{i \theta_{n}}, w_{n}=r_{n} e^{i \lambda_{n}}$ satisfying $\left|\theta_{n}-\omega\left(r_{n}\right)\right| \leq \delta$ and $\left|\lambda_{n}-\omega\left(r_{n}\right)\right| \leq \delta$ then

$$
\limsup _{r \rightarrow \infty} b(r) \geq 2\left(A_{1}-\frac{8}{\alpha_{0}^{2}} \log \left|\operatorname{sn}\left(\frac{K^{\prime} \delta}{\pi}, k^{\prime}\right)\right|\right) \text {. }
$$

(iii) If $\limsup _{r \rightarrow \infty} b(r)=2 A_{1}$ then all but a finite number of the multiple zeros of $f$ must satisfy $\left|\theta_{n}-\omega\left(r_{n}\right)\right|=\pi$.

\section{AN AUXILIARY LEMMA}

The principle of our proofs is the same as that of Hayman's [3], but instead of using the cotangent function we use theta functions. This leads to the fol- 
lowing lemma which is fundamental to our proofs and which may also be of independent interest.

Lemma. Let $f(z)$ be regular in the annulus $D\left(r_{0}, \alpha_{0}\right)$ and let $L(0)=\log \sqrt{M\left(r_{0} e^{-\alpha_{0} / 2}\right) M\left(r_{0} e^{\alpha_{0} / 2}\right)}$.

(i) If $f\left(z_{1}\right)=0$ for some point $z_{1}$ satisfying $\left|z_{1}\right|=r_{0}$, then

$$
\left|f\left(z_{1} e^{i \tau}\right)\right| \leq \exp (L(0)) \cdot k^{\prime \frac{1}{2}}\left|\operatorname{sn}\left(\frac{K^{\prime} \tau}{\pi}, k^{\prime}\right)\right|
$$

for all real $\tau$.

(ii) Suppose $f\left(z_{1}\right)=f\left(z_{2}\right)=0$ where $z_{1}=r_{0} e^{i \theta_{1}}, z_{2}=r_{0} e^{i \theta_{2}}$. Set $z_{0}=$ $r_{0} e^{i \gamma}$ where $\gamma=\frac{1}{2}\left(\theta_{1}+\theta_{2}\right)$. Then, if $\beta=\frac{1}{2}\left(\theta_{2}-\theta_{1}\right)$ and $-\pi \leq \tau \leq \pi$, we have

$$
\left|f\left(z_{0} e^{i \tau}\right)\right| \leq \exp (L(0)) \cdot k^{\prime}\left|\operatorname{sn}\left(\frac{K^{\prime}(\tau-\beta)}{\pi}, k^{\prime}\right) \operatorname{sn}\left(\frac{K^{\prime}(\tau+\beta)}{\pi}, k^{\prime}\right)\right| .
$$

It will be convenient to omit the subscript in $\alpha_{0}$ and write $\alpha$ instead. Let $S=S(\alpha)$ be the vertical strip in the $z$-plane defined by

$$
S=\left\{z=\sigma+i \tau:-\frac{1}{2} \alpha<\sigma<\frac{1}{2} \alpha,-\infty<\tau<+\infty\right\} .
$$

The maximum principle will be applied to a certain auxiliary function on $S$ and this requires a study of factors of the form $\left(1 \pm q^{2 n} e^{ \pm i \pi z / \alpha}\right)$ on the boundary of $S$. More precisely, if $q=e^{-\pi^{2} / \alpha}$ where $\alpha>0$ and $z= \pm \frac{1}{2} \alpha+i y$ then, since $e^{ \pm i \pi z / \alpha}$ is pure imaginary, we have

$$
\left|\frac{1+q^{2 n} e^{i \pi z / \alpha}}{1-q^{2 n} e^{i \pi z / \alpha}}\right|=\left|\frac{1+q^{2 n} e^{-i \pi z / \alpha}}{1-q^{2 n} e^{-i \pi z / \alpha}}\right|=1 \quad(\infty<y<+\infty) .
$$

Also, if $z= \pm \frac{1}{2} \alpha+i y$ then

$$
|\cot (\pi z / 2 \alpha)|=1 \quad(-\infty<y<+\infty) .
$$

It is clear for each fixed $n$ that if $z \in S$ and $z \rightarrow \infty$ then

$$
\left|\frac{1+q^{2 n} e^{ \pm \pi i z / \alpha}}{1-q^{2 n} e^{ \pm \pi i z / \alpha}}\right| \rightarrow 1
$$

Finally, since

$$
\frac{\sinh ^{2} \pi y / 2 \alpha}{1+\sinh ^{2} \pi y / 2 \alpha} \leq\left|\cot \left(\frac{\pi x}{2 \alpha}+i \frac{\pi y}{2 \alpha}\right)\right|^{2} \leq \frac{1+\sinh ^{2} \pi y / 2 \alpha}{\sinh ^{2} \pi y / 2 \alpha}
$$

for $|x| \leq \frac{1}{2} \alpha$ and $y \neq 0$, we see that $|\cot (\pi z / 2 \alpha)| \rightarrow 1$ as $z \rightarrow \infty$ inside $S$.

We are now ready to proceed with the proof of the lemma.

Proof. We shall only prove (3.2) since the proof of (3.1) is similar (and simpler). Let $f$ satisfy the conditions in (ii) of the lemma and consider the function $f\left(z_{0} e^{z}\right)$ on the strip $S$. Let $L(\sigma)=B \sigma+C$ where the real constants $B$ and $C$ are determined by the equations

$$
\lim _{\sigma \rightarrow \pm \frac{1}{2} \alpha}\left(\log M\left(r_{0} e^{\sigma}\right)-L(\sigma)\right)=0 .
$$


Since $\log M\left(r_{0} e^{\sigma}\right)$ is a bounded convex function of $\sigma$ in the interval $I\left(r_{0}, \alpha\right)$, the limits exist and determine $L$ uniquely. By the Hadamard three-circle theorem we also have

$$
\left|f\left(z_{0} e^{z}\right)\right| \leq \exp (L(\sigma))
$$

for all $z=\sigma+i \tau$ in $S$.

Fix the integer $N \geq 1$ and consider in $S$ the functions

$$
\psi_{N}(z)=\frac{\cos (\pi z / 2 \alpha)}{\sin (\pi z / 2 \alpha)} \prod_{n=1}^{N} \frac{\left(1+q^{2 n} e^{i \pi z / \alpha}\right)\left(1+q^{2 n} e^{-i \pi z / \alpha}\right)}{\left(1-q^{2 n} e^{i \pi z / \alpha}\right)\left(1-q^{2 n} e^{-i \pi z / \alpha}\right)}
$$

and

$$
\phi_{N}(z)=f\left(z_{0} e^{z}\right) e^{-L(z)} \psi_{N}(z-i \beta) \psi_{N}(z+i \beta)
$$

where $\beta=\frac{1}{2}\left(\arg z_{2}-\arg z_{1}\right)$ may be assumed to satisfy $0 \leq \beta<\pi$. Suppose $\beta \neq 0$. The zeros of the denominator of $\phi_{N}$ in $S$ are at the zeros of $\sin (\pi(z-i \beta) / 2 \alpha) \sin (\pi(z+i \beta) / 2 \alpha)$ in $S$, which are $\pm i \beta$, and at the zeros of the factors $\left(1-q^{2 n} e^{i \pi(z \pm i \beta) / \alpha}\right)\left(1-q^{2 n} e^{-i \pi(z \pm i \beta) / \alpha}\right)$ in $S$, which are $\pm i \beta \pm 2 n \pi i$. So the zeros of the denominator of $\phi_{N}$ in $S$ occur at $\pm i \beta+2 n \pi i$ where $n=0, \pm 1, \pm 2, \ldots, \pm N$. On the other hand, since $z_{0}=r_{0} e^{\frac{i}{2}\left(\theta_{1}+\theta_{2}\right)}$ and $f\left(r_{0} e^{i \theta_{1}}\right)=f\left(r_{0} e^{i \theta_{2}}\right)=0$, the function $f\left(z_{0} e^{z}\right)$ has zeros at all the points $\pm i \beta+2 n \pi i$ where $n=0, \pm 1, \pm 2, \ldots$. Thus the function $\phi_{N}$ is regular in $S$. If $\beta=0$ then the zeros of the denominator of $\phi_{N}$ occur at $2 n \pi i$ and $f$ has double zeros there so that $\phi_{N}$ is again regular in $S$. The boundary behavior of $\phi_{N}$ follows immediately from that of $\psi_{N}$. Thus $\lim \sup \left|\phi_{N}(z)\right| \leq 1$ as $z$ approaches any finite boundary point of $S$ by (3.4) and (3.5). Also lim sup $\left|\phi_{N}(z)\right| \leq 1$ as $z \rightarrow \infty$ inside $S$ by (3.6) and (3.7). It now follows by the maximum principle that $\left|\phi_{N}(z)\right| \leq 1$ for all $z \in S$ and so by (3.8)

$$
\left|f\left(z_{0} e^{z}\right)\right| \leq \exp (L(\sigma))\left|\psi_{N}(z-i \beta) \psi_{N}(z+i \beta)\right|^{-1}
$$

where $z=\sigma+i \tau,|\sigma| \leq \frac{1}{2} \alpha$ and $-\infty<\tau<\infty$.

If we let $N \rightarrow \infty$ the product defining $\psi_{N}$ becomes a ratio of theta functions given in (5). These can then be simplified using (6), (8) and (9) to give by (3.11)

$$
\left|f\left(z_{0} e^{z}\right)\right| \leq \exp (L(\sigma)) k^{\prime}\left|\frac{s n}{c n}\left(\frac{K(z-i \beta)}{\alpha}, k\right)\right|\left|\frac{s n}{c n}\left(\frac{K(z+i \beta)}{\alpha}, k\right)\right|,
$$

valid for all $z=\sigma+i \tau,|\sigma| \leq \frac{1}{2} \alpha$ and $-\infty<\tau<\infty$.

In particular, if we take $\sigma=0$ and use the Jacobian imaginary transformation $[6$, p. 506] namely,

we obtain

$$
s n(i u, k)=i \frac{s n\left(u, k^{\prime}\right)}{c n\left(u, k^{\prime}\right)}, \quad c n(i u, k)=\frac{1}{c n\left(u, k^{\prime}\right)},
$$

$$
\left|f\left(z_{0} e^{i \tau}\right)\right| \leq \exp (L(0)) \cdot k^{\prime}\left|\operatorname{sn}\left(K(\tau-\beta) / \alpha, k^{\prime}\right)\right|\left|\operatorname{sn}\left(K(\tau+\beta) / \alpha, k^{\prime}\right)\right| .
$$

If we now recall that $q=e^{-\pi^{2} / \alpha}$ and, from (8), $\frac{\pi K^{\prime}}{K}=\log q^{-1}$, we obtain $\frac{K^{\prime}}{K}=\frac{\pi}{\alpha}$ so that $\frac{K(\tau-\beta)}{\alpha}=\frac{K^{\prime}(\tau-\beta)}{\pi}$ and $\frac{K(\tau+\beta)}{\tau}=\frac{K^{\prime}(\tau+\beta)}{\pi}$. Thus (3.13) takes the form

$$
\left|f\left(z_{0} e^{i \tau}\right)\right| \leq \exp (L(0)) \cdot k^{\prime}\left|s n\left(\frac{K^{\prime}(\tau-\beta)}{\pi}, k^{\prime}\right) \operatorname{sn}\left(\frac{K^{\prime}(\tau+\beta)}{\pi}, k^{\prime}\right)\right| .
$$

This finishes the proof of the lemma. 


\section{Proof of Theorem 3}

Let us prove part (ii) of Theorem 3 since the proof of part (i) is similar. Suppose then that $0<\delta \leq \pi$ and that $f$ has infinitely many pairs of zeros $z_{n}=r_{n} e^{i \theta_{n}}$ and $w_{n}=r_{n} e^{i \lambda_{n}}$ having equal moduli and satisfying

$$
\left|\theta_{n}-\omega\left(r_{n}\right)\right| \leq \delta \text { and }\left|\lambda_{n}-\omega\left(r_{n}\right)\right| \leq \delta
$$

where $\left|f\left(r_{n} e^{i \omega\left(r_{n}\right)}\right)\right|=M\left(r_{n}\right)$.

Let $L_{n}(\sigma)=B_{n} \sigma+C_{n}$ where the real constants are, as in the proof of the lemma, determined by the equations

$$
\lim _{\sigma \rightarrow \pm \frac{1}{2} \alpha}\left(\log M\left(r_{n} e^{\sigma}\right)-L_{n}(\sigma)\right)=0 .
$$

Applying part (ii) of the lemma with $z_{0}=r_{n} e^{i\left(\theta_{n}+\lambda_{n}\right) / 2}, z_{1}=z_{n}, z_{2}=w_{n}$ and $\tau=\omega\left(r_{n}\right)-\left(\theta_{n}+\lambda_{n}\right) / 2$ we obtain

$$
M\left(r_{n}\right) \leq \exp \left(L_{n}(0)\right) k^{\prime}\left|\operatorname{sn}\left(\frac{K^{\prime}(\tau-\beta)}{\pi}, k^{\prime}\right) s n\left(\frac{K^{\prime}(\tau+\beta)}{\pi}, k^{\prime}\right)\right| .
$$

Here $\beta=\frac{1}{2}\left(\lambda_{n}-\theta_{n}\right)$ and so, in view of (4.1), we have

$$
|\tau-\beta| \leq \delta \text { and }|\tau+\beta| \leq \delta .
$$

Since $\operatorname{sn}\left(u, k^{\prime}\right)$ is an odd function of $u$ that increases on $0 \leq u \leq K^{\prime}$ we have

$$
\left|s n\left(\frac{K^{\prime}(\tau-\beta)}{\pi}, k^{\prime}\right) s n\left(\frac{K^{\prime}(\tau+\beta)}{\pi}, k^{\prime}\right)\right| \leq\left|s n\left(\frac{K^{\prime} \delta}{\pi}, k^{\prime}\right)\right|\left|s n\left(\frac{K^{\prime} \delta}{\pi}, k^{\prime}\right)\right| \text {, }
$$

and so

$$
\log M\left(r_{n}\right) \leq L_{n}(0)+\log k^{\prime}+2 \log \left|\operatorname{sn}\left(\frac{K^{\prime} \delta}{\pi}, k^{\prime}\right)\right| .
$$

Now put $g(\sigma)=L_{n}(\sigma)-\log M\left(r_{n} e^{\sigma}\right)+\frac{A}{2}\left(\sigma^{2}-\frac{1}{4} \alpha^{2}\right)$ and assume that $b(r)<A$ for all $r \in I\left(r_{n}, \alpha\right)$. Then $g^{\prime \prime}(\sigma)=-b\left(r_{n} e^{\sigma}\right)+A$ so that the assumption on $b$ implies that $g$ is convex on $\left[-\frac{1}{2} \alpha, \frac{1}{2} \alpha\right]$. As $g\left(-\frac{1}{2} \alpha\right)=g\left(\frac{1}{2} \alpha\right)=0$, it follows that $g(\sigma) \leq 0$ for all $|\sigma| \leq \frac{1}{2} \alpha$. In particular $g(0) \leq 0$ and so

$$
\log M\left(r_{n}\right) \geq L_{n}(0)-A \frac{\alpha^{2}}{8} .
$$

If this last inequality is combined with (4.5) we obtain

$$
A \geq \frac{-8}{\alpha^{2}}\left(\log k^{\prime}+2 \log \left|\operatorname{sn}\left(\frac{K^{\prime} \delta}{\pi}, k^{\prime}\right)\right|\right) \text {. }
$$

It remains to recall, from (11) and (12), the definition $A_{1}=\frac{-8}{\alpha_{0}^{2}} \log k^{1 / 2}$ to see that inequality (2.4) of Theorem 3 follows from (4.6) and the assumption of an infinitude of pairs of zeros having equal moduli. This finishes the proof of part (ii) of Theorem 3 and we have, in passing, proved part (ii) of Theorem 1. Indeed, taking $\delta=\pi$ in (4.6) gives the required inequality in part (ii) of Theorem 1 and, of course, (4.1) is always satisfied in this case. The proofs of part (i) of Theorem 3 and part (i) of Theorem 1 are similar and use (3.1) 
instead of (3.2). To prove part (iii) of Theorem 3, note that the assumption $\lim \sup b(r)=2 A_{1}$ implies that $s n\left(\frac{K^{\prime} \delta}{\pi}, k^{\prime}\right)=1$ and so $\delta=\pi$. Thus, either $f$ has only a finite number of multiple zeros, in which case (2.4) does not apply; or $f$ has an infinite number of multiple zeros $z_{n}=r_{n} e^{i \theta_{n}}$ in which case (2.4) applies and gives $\left|\theta_{n}-\omega\left(r_{n}\right)\right|=\pi$ for all large $n$ as required. This finishes the proof of Theorem 3 .

\section{Proofs (CONTINUED)}

The inequalities (2.1) and (2.2) are immediate consequences of parts (i) and (ii) of Theorem 1. To prove part (i) of Corollary 2 observe that if the coefficients of $f$ are real then complex zeros, when they exist, occur in conjugate pairs. So if $f$ has real coefficients and an infinite number of complex zeros then part (ii) of Theorem 1 or Theorem 3 implies that $\limsup _{r \rightarrow \infty} b(r) \geq 2 A_{1}$, contradicting (2.2). Thus $f$ can have at most a finite number of complex zeros and all other zeros must be real and simple. Part (ii) of Corollary 2 follows from part (i) and the fact that, since the coefficients of $f$ are nonnegative, the real zeros of $f$ when they occur cannot be positive.

Proof of Theorem 2. Let $f$ be entire with nonnegative Maclaurin coefficients and suppse that $\limsup b(r)=\frac{1}{4}$. Since $2 A_{1}>0.365>0.25$, Corollary 2 implies that, except for a possible finite number, all the zeros of $f$ are simple, real and negative. Thus we may write

$$
f(z)=p(z) \prod_{n=1}^{\infty}\left(1+z / r_{n}\right)
$$

where $p$ is a polynomial carrying the nonreal zeros of $f$ and the product carries all the negative real and simple zeros $\left\{-r_{n}\right\}$ of $f$. Since we are given that all coefficients of $f$ are nonnegative, we have $M(r, f)=f(r)$ and so

$$
b(r, f)=d^{2} \log p(r) / d(\log r)^{2}+\sum_{n=1}^{\infty} \frac{r r_{n}}{\left(r+r_{n}\right)^{2}} .
$$

The first term on the right-hand side of (5.2) tends to zero as $r \rightarrow \infty$ and so does not contribute to $\lim \sup b(r)$. Thus we have

$$
\frac{1}{4}=\limsup _{r \rightarrow \infty} b(r) \geq \limsup _{n \rightarrow \infty} b\left(r_{n}\right) \geq \limsup _{n \rightarrow \infty}\left(\frac{1}{4}+\frac{r_{n} r_{n+1}}{\left(r_{n}+r_{n+1}\right)^{2}}\right) \geq \frac{1}{4} .
$$

Therefore

$$
\limsup _{n \rightarrow \infty} \frac{r_{n+1} / r_{n}}{\left(1+r_{n+1} / r_{n}\right)^{2}}=0 \text {. }
$$

If a subsequence $r_{n_{k}+1} / r_{n_{k}}$ of $r_{n+1} / r_{n}$ were bounded (above), say by $M$, we would contradict (5.3). Hence $r_{n+1} / r_{n} \rightarrow \infty$ as $n \rightarrow \infty$. Conversely, if the real simple zeros of $f$ satisfy $r_{n+1} / r_{n} \rightarrow \infty$ as $n \rightarrow \infty$ and $b(r)$ is bounded, then $f$ factors as in (5.1) and $b(r, f)$ is given by (5.2) where, again, only the second term on the right-hand side matters. We can write

$$
\sum_{n=1}^{\infty} \frac{r r_{n}}{\left(r+r_{n}\right)^{2}} \leq \frac{r r_{n}}{\left(r+r_{n}\right)^{2}}+\frac{1}{r} \sum_{k=1}^{n-1} r_{k}+r \sum_{k=n+1}^{\infty} 1 / r_{k}
$$


We may assume that $r_{n+1} / r_{n}>M>1$ for all $n \geq 1$. Then we have

$$
\frac{r r_{n}}{\left(r+r_{n}\right)^{2}} \leq \sum_{n=1}^{\infty} \frac{r r_{n}}{\left(r+r_{n}\right)^{2}} \leq \frac{r r_{n}}{\left(r+r_{n}\right)^{2}}+\frac{M}{M-1}\left\{\frac{r_{n-1}}{r}+\frac{r}{r_{n+1}}\right\} \text {. }
$$

By considering $r \in\left(\left(r_{n-1} r_{n}\right)^{1 / 2},\left(r_{n} r_{n+1}\right)^{1 / 2}\right)$, we conclude $\limsup _{r \rightarrow \infty} b(r)=\frac{1}{4}$ as required.

\section{ACKNOWLEDGMENT}

I wish to thank Alexandre Eremenko for his interest in this work; in particular, the question whether (3) follows from (2) was raised by him. I am also grateful to David Drasin for reading through the manuscript and suggesting some editorial changes. This work was completed during a sabbatical leave at Purdue University. The hospitality of the Mathematics Department at Purdue is gratefully acknowledged.

\section{REFERENCES}

1. F. F. Abi-Khuzam, Maximum modulus convexity and the location of zeros of an entire function, Proc. Amer. Math. Soc. 106 (1989), 1063-1068.

2. V. S. Boichuk and A. A. Gol'dberg, The three-lines theorem, Mat. Zametki 15 (1974), 45-53. (Russian)

3. W. K. Hayman, Note on Hadamard's convexity theorem, Entire Functions and Related Parts of Analysis, Proc. Sympos. Pure Math., vol. 11, Amer. Math. Soc., Providence, RI, 1968, pp. 210-213.

4. B. Kjelleberg, The convexity theorem of Hadamard-Hayman, Proc. Sympos. Math., Stockholm (June 1973, Royal Institute of Technology), pp. 87-114.

5. __ Review of 1, Zentralblatt für Math., 1990.

6. E. T. Whittaker and G. N. Watson, A course of modern analysis, Cambridge Univ. Press, Cambridge, 1980.

Department of Mathematics, American University of Beirut, Beirut, Lebanon

Current address: Department of Mathematical Sciences, Purdue University, West Lafayette, Indiana 47907

E-mail address: farukakh@layla.aub.ac.lb 